\title{
Modelización de la cohabitación de hogares en la informalidad urbana*
}

\section{Modeling Household Cobabitation in Urban Informality Modelagem de coabitação familiar na informalidade urbana}

\author{
Diva Marcela García García ** \\ Juan Antonio Módenes ${ }^{* *}$
}

Recibido: 23 de noviembre de 2020

Aprobado: 25 de marzo de 2021

https://doi.org/10.12804/revistas.urosario.edu.co/territorios/a.9943

Para citar este artículo

García García, D. M., \& Módenes, J. A. (2021). Modelización de la cohabitación de hogares en la informalidad urbana. Territorios, (45), 167-187. https://doi.org/10.12804/revistas.urosario.edu.co/ territorios/a.9943

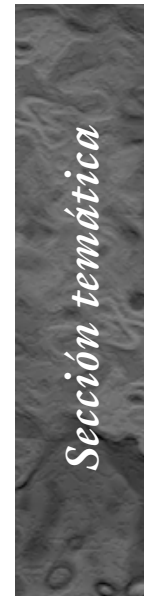

* El presente artículo se deriva de la tesis doctoral "Convergencias y divergencias sociodemográficas en el sistema residencial bogotano" en el programa de Doctorado en Demografía de la Universitat Autónoma de Barcelona y el Centre d'Estudis Demografics (2019).

** Investigadora y docente Universidad Sergio Arboleda, Bogotá. Correo electrónico:diva.garcia@ usa.edu.co ORCID: https:// orcid.org/0000-00024773-6897

*** Investigador y docente Universitat Autónoma de Barcelona. Correo electrónico:jamodenes@ced. uab.es ORCID: https:// orcid.org/Orcid: 00000003-3049-7495 
Palabras clave

Cohabitación; informalidad urbana; regresión logistica; hogares; déficit.

Keywords

Cobabitation; urban informality; logistic regression; households; deficit.

Palavras-chave

Coabitação; informalidade urbana; regressão logística; lares; déficit.

territarias 45

\section{RESUMEN}

El trabajo demuestra que, en Bogotá, la informalidad urbana, que permite alta mutabilidad de la infraestructura residencial, al interactuar con prácticas familiares tradicionales y necesarias en los grupos populares, genera una alta complejidad en las formas de organización doméstica que se mantiene a pesar de los procesos de legalización. Se profundiza en la cohabitación (más de un hogar por vivienda), buscando establecer su relación estadística con características individuales, del hogar y la vivienda, y biográficas del barrio. Para ello, se realizan dos modelos de regresión logística multivariante: uno para todos los sectores censales clasificados como de bajo nivel social y otro comparando en dichos sectores, los de origen formal e informal. Se armonizó información censal de tipo demográfico, con información espacial que permitió la clasificación del área urbana en función de su nivel social y de su origen urbanístico, la cual se incluyó como variable independiente.

\section{ABSTRACT}

The work shows that in Bogotá, urban informality, which allows high mutability of the residential infrastructure, by interacting with traditional and necessary family practices in popular groups, generates a high complexity in the forms of domestic organization that is maintained despite the legalization processes. Cohabitation (more than one household per dwelling) is studied in depth, seeking to establish its statistical relationship with individual, home and dwelling characteristics, and biographical characteristics of the neighborhood. To do this, two multivariate logistic regression models are carried out: one for all census sectors classified as low social level, and another comparing in these sectors, those of formal and informal origin. To this aim, demographic census information was harmonized with spatial information that allowed the classification of the urban area based on its social level and urban origin, which was included as an independent variable.

\section{RESUMO}

O trabalho mostra que, em Bogotá, a informalidade urbana, que permite alta mutabilidade da infraestrutura residencial, ao interagir com práticas familiares tradicionais e necessárias em grupos populares, gera uma alta complexidade nas formas de organização doméstica que se mantém apesar dos processos de legalização. A coabitação (mais de um lar por domicílio) é estudada em profundidade, buscando estabelecer sua relação estatística com as características individuais, do lar e do domicílio, e com as características biográficas do bairro. Para tanto, são realizados dois modelos de regressão logística multivariada: um para todos os setores censitários classificados como de baixo nível social, e outro comparando, nesses setores, os de origem formal e informal. Para isso, as informações censitárias demográficas foram harmonizadas com as informações espaciais que permitiram classificar a área urbana com base no seu nível social e origem urbana, a qual foi incluída como variável independente. 


\section{Introducción}

Durante las décadas pasadas, como consecuencia de los modos de producción capitalista, que en Colombia estuvieron atravesados por condiciones de violencia, América Latina vivió un intenso flujo migratorio hacia las ciudades, aumentando la demanda de bienes y servicios. El desajuste de dicha demanda con los sistemas de acceso a la vivienda, altamente excluyentes para la población de menores ingresos, e incompatibles con el empleo informal, generó una demanda residencial que solo pudo ser resuelta en el mercado informal. ${ }^{1}$

Aunque con el paso del tiempo buena parte de estas zonas han tenido intervenciones estatales, muchas de las condiciones iniciales del hábitat se mantienen y otras se transforman a partir del uso de sus residentes.

Así, se ha constituido históricamente un mercado inmobiliario peculiar, con unas condiciones institucionales y físicas que son el escenario de la compleja relación entre demanda y oferta residencial. Sobre esa base, la pregunta general de este trabajo se refiere al efecto que ejerce la urbanización informal sobre las pautas de corresidencia, la cual opera bajo una lógica de producción residencial basada en esquemas de autoconstrucción y desarrollo progresivo. Se quiere indagar si estas condiciones, que permiten alto dinamismo y mutabilidad de la infraestructura producida, al interactuar con prácticas familiares tradicionales $-\mathrm{y}$ necesariasen los grupos populares, generan una alta complejidad en las formas de organización doméstica.

Tomando al allegamiento externo o cohabitación (existencia de dos o más hogares dentro de un domicilio), como ejemplo de la complejidad residencial que se aloja en el hábitat producido informalmente, se desarrollan dos preguntas: 1) ¿Hay un efecto neto del origen urbanístico en el allegamiento externo, independientemente de la estructura sociodemográfica, o se trata precisamente de un efecto de composición, porque la informalidad atrae población más propensa a la complejidad familiar? y 2) ¿Hay efectos diferenciados de las variables que explican el allegamiento externo en cada zona de origen urbanístico? Al interior de esta última pregunta, se aloja una tercera, referida a la permanencia o mutabilidad de estas prácticas de cohabitación en función de la transformación de las condiciones de informalidad.

\section{Informalidad urbana $y$ cohabitación: aproximación y antecedentes}

Una de las particularidades de la producción informal de la ciudad es que la construcción de la vivienda sucede al margen de requisitos legales, a manos del hogar que será el posterior residente. La vivienda en autoconstrucción puede dar inicio a la vivienda progresiva, entendida como un
Según Abramo (2012), la urbanización informal se entiende como un conjunto de irregularidades de tipo urbanistico, constructivo y en relación con el derecho de propiedad. territarios 45 169 
${ }^{2}$ Se entiende el hogar como una forma de agrupamiento de los individuos que se reúnen en un mismo lugar para vivir cotidianamente durante algún tiempo, incluyendo una economía compartida, una domesticidad colectiva (Jelin, 2005). El curso de vida de los hogares da cuenta principalmente de aquellos que presentan lazos familiares y que revelan un momento especifico de conformación, en función de la edad de sus integrantes y de la relación de dependencia entre ellos (Ullman \& Valera, 2014).

\section{territarias 45}

proceso constructivo que se desarrolla a lo largo del tiempo y que está sujeta a cambios y variaciones en su estructura (Gelabert \& González, 2013). Esta forma de producción de la vivienda guarda una relación estrecha con el momento del curso de vida de los hogares ${ }^{2}$ y sus miembros, el cual determina las necesidades de espacio y los usos de los mismos, así como las posibilidades de inversión en el proceso constructivo:

Cuando se trata de una pareja joven recién formada, lanzan la autoconstrucción y en el período más productivo y a medida que los hijos se multiplican y crecen, se va expandiendo la casa. Cuando los hijos ya han crecido y los padres entran en una fase en la que les es más difícil obtener ingresos laborales, el espacio desocupado por los hijos que abandonan el hogar puede ser alquilado, de manera que el arrendamiento complementa sus ingresos. (Jaramillo, 2008 , p. 29)

Además de lo anterior, que resulta clave para comprender la cohabitación en la informalidad, otra característica fundamental de este tipo de vivienda es la posibilidad de obtener ingresos monetarios a partir de la construcción o destinación de ciertos espacios para usos económicos, tales como habitaciones o locales comerciales que se alquilan, y conforman un submercado del arriendo (Jaramillo, 2008).
Así, un perfil específico de población en términos socioeconómicos y de pasado rural, se aloja en un mercado inmobiliario conformado por un tipo de vivienda flexible y mutable, que resulta atractiva e incluso "conveniente" (Camargo \& Hurtado, 2012; Gelabert \& González, 2013), para las formas residenciales y familiares identificadas en los sectores populares.

En este último sentido, cabe recordar que entre más se desciende en la jerarquía social, más decisivo es el papel de la familia en las prácticas residenciales (Bonvalet \& Dureau, 2002), dado que las redes de apoyo que allí se localizan resultan fundamentales para la subsistencia y para el acceso a bienes y servicios escasos y costosos en el mercado, como la vivienda. En este contexto, familia, vecinos y amigos facilitan el establecimiento en un nuevo entorno, la construcción de las viviendas o la recepción de hogares o personas que requieran de un lugar para vivir (Di Virgilio \& Gil, 2012). Es así como las solidaridades familiares muchas veces se expresan en la cohabitación o el allegamiento externo (Bonvalet \& Dureau, 2002), generalmente intergeneracional, que puede ser visto como una “estrategia del grupo familiar mientras se reúne el aporte personal para comprar una vivienda" (Bonvalet \& Dureau, 2002, p. 87). Se basa en el uso de relaciones de cooperación, que producen una solución a problemas materiales a través de la complementariedad de roles al interior de los 
hogares (Urrutia et al., 2016). Sin embargo, no se puede idealizar la cohabitación como una red de apoyo, pues también es fuente de conflicto (González, 2001).

De ahí que, en el contexto latinoamericano, el allegamiento externo o cohabitación sea uno de los indicadores del déficit habitacional cuantitativo, que refleja las necesidades de producción residencial de una sociedad (UN-Hábitat, 2004). Su principal afectación a la calidad de vida de la población se asocia con la falta de intimidad de los hogares independientes, que no pueden contar con una vivienda exclusiva, lo que impide el cumplimiento de una de las funciones sociales de la vivienda.

Además de complejizar numéricamente la relación entre hogares y viviendas, y desafiar el paradigma explícito de que a cada hogar corresponde una vivienda, el allegamiento externo pone en debate algunos de los supuestos culturales que rigen la relación entre ambos, como, por ejemplo, la necesidad de una vivienda como condición necesaria para la emancipación, o para la conformación de hogares en diferentes momentos de la vida.

Estudiar estas prácticas residenciales en contextos de origen informal permite indagar por la forma en que los mecanismos de producción de ciudad tienen efectos en el nivel micro del fenómeno residencial y su impacto en las formas de heredar, convivir o gestionar la vivienda. Además, interesa conocer si las prácticas de cohabitación localizadas en la informalidad y acentuadas por su forma de producción urbana, (hipótesis que sigue este trabajo) se transforman y "formalizan" con el paso del tiempo y con las intervenciones de los actores privados, de la población y del Estado. Este último actor ha encaminado su función a disponer los mecanismos procesales y legales para que las condiciones de los barrios de origen informal se ajusten a los mínimos que la formalidad demanda, solventando a través de diferentes estrategias de política pública las consecuencias del fenómeno, asociadas al aislamiento y la marginalidad (López, 2017). Las principales intervenciones estatales son los procesos de legalización y de mejoramiento de barrios, que pueden incluir iniciativas para el mejoramiento de las condiciones físicas de la vivienda, de los espacios públicos, vías e infraestructura, así como programas de saneamiento de la tenencia. Sin embargo, no todas ellas se realizan conjuntamente por lo que se ha documentado su impacto diferenciado en la calidad de vida de los habitantes (Torres, 2012). Este interrogante sobre la transformación implica repensar la manera dicotómica en que se ha estudiado el fenómeno, pues la situación actual de los territorios que surgieron informalmente, no parece ajustarse ni a una ni a otra categoría y los límites que separan "lo formal" de "lo informal" son cada vez más difusos. 
Figura 1. Asentamientos según origen urbanístico. Bogotá, 2008

Nivel socioeconómico bajo

Asentamientos legalizados

Asentamientos informales

Asentamientos formales
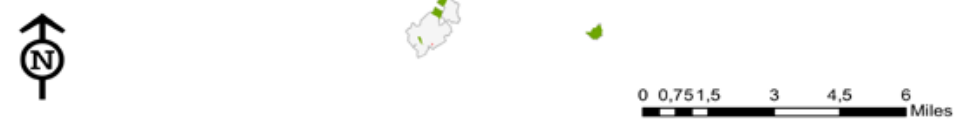

Fuente: elaboración propia con base en información de la Secretaría de Planeación de Bogotá (2008) y la Fundación Techo (2015).

\section{Metodología}

\section{Fuentes de datos y \\ clasificación de zonas}

Con base en la información cartográfica de la base de datos de la Secretaría de Planeación de Bogotá, que contiene los datos sobre los barrios legalizados, en trámite de legalización o con legalización denegada hasta 2008, se clasificó el área urbana de Bogotá en tres categorías según su origen (figura 1):

a. Asentamientos formales: que nacieron formales; nunca han sido objeto de procesos de legalización.

b. Asentamientos legalizados: surgieron informalmente y fueron legalizados por el distrito.

c. Asentamientos informales: esta categoría reúne barrios de vieja data, que no han sido, ni serán legalizados, especialmente por ubicarse en zonas con restricciones para la urbanización. Reúne además los desarrollos informales más recientes, cuya legalización está en proceso. Se complementó la información de esta categoría con la base de datos construida por la ONG Techo (2015), con el fin de incorporar posibles asentamientos que no hubieran iniciado procesos administrativos.

La clasificación de cada polígono se aplicó a cada hogar, armonizando los datos censales de 2005 a partir del

\section{territarias 45}


identificador de la manzana catastral de residencia. A pesar de lo antiguo de dichos datos, son los que se encontraban totalmente disponibles a nivel de manzana en el momento de elaboración de esta investigación, que se constituye en un punto de partida para futuras comparaciones con datos más recientes. La distribución de hogares resultante entre las categorías revela que, para 2005 , el $62 \%$ se localizaron en áreas formales, mientras que el $36,1 \%$ lo hicieron en áreas legalizadas y el 1,9\% residía en zonas informales.

Para aislar el efecto del nivel socioeconómico de los sectores urbanos, el análisis se limitó a los hogares residentes en las zonas clasificadas en el nivel social bajo según el Índice de Condiciones de Vida (ICV) calculado en Mayorga et al. (2019).

\section{Modelos de regresión logística}

Se utilizó la regresión logística multivariante, que permite estudiar la relación entre una variable dependiente categórica y varias independientes. En este caso, la variable dependiente dicotómica es la condición de un hogar, de compartir su vivienda con otro (1) o no (0) (se incluyen como cohabitantes los hogares unipersonales).

Se plantearon tres modelos, para los que previamente se hicieron pruebas de multicolinealidad e interacción entre las variables independientes. Para el desarrollo de las regresiones se utilizó primero el método stepwise en el que las variables independientes son incorporadas en pasos sucesivos $\mathrm{y}$, a medida que se introducen, se predice la variable dependiente de forma más precisa, aunque la precisión que añade cada variable adicional es sucesivamente menor. Las variables se incluyen mientras su nivel de significación estadística del estadístico -2 Log Likelihood (en adelante-2LL) se encuentra en los límites preestablecidos como criterio de inclusión de predictores, excluyéndolas si dejan de cumplir este criterio (Jovell, 1995). En la presentación de cada modelo se incluyen los niveles de ganancia relativa de una variable sobre la anterior. Posteriormente, las variables seleccionadas se vincularon al modelo para el cálculo de las odds ratio de las categorías de cada variable, que dan cuenta de la propensión a la ocurrencia del fenómeno.

A continuación, se detallan las variables independientes y el planteamiento de cada modelo, en función de la pregunta de investigación a la que responde:

\section{Relación entre el allegamiento externo y el origen urbanistico: ¿efecto neto o de composición?}

Para el abordaje de estas preguntas se realizaron los siguientes modelos:

Modelo 0: se utiliza como única variable independiente el origen de urbanización del sector en que residen los hogares (formal, legalizado e informal). 
Modelo 1: pretende controlar la propensión a la cohabitación de los hogares por un conjunto de variables de diferentes niveles de análisis, tal como se muestra en la tabla 1.

Tabla 1. Características de las variables incluidas en el modelo 1

\begin{tabular}{|c|c|c|}
\hline $\begin{array}{l}\text { Nivel/tipo } \\
\text { de variables }\end{array}$ & Variables & Fuente \\
\hline $\begin{array}{l}\text { Individuales } \\
\text { del jefe del } \\
\text { hogar }\end{array}$ & $\begin{array}{l}\text { - Sexo } \\
\text { - Edad } \\
\text { - Nivel educativo } \\
\text { - Lugar de } \\
\text { nacimiento fuera } \\
\text { de Bogotá }\end{array}$ & \multirow[t]{2}{*}{$\begin{array}{l}\text { Microdatos } \\
\text { Censo } 2005\end{array}$} \\
\hline $\begin{array}{l}\text { Vivienda/ } \\
\text { Hogar }\end{array}$ & $\begin{array}{l}\text { - Tipo de hogar } \\
\text { - Tipo de vivienda }\end{array}$ & \\
\hline Barrial & $\begin{array}{l}\text { - Década de } \\
\text { desarrollo urbano } \\
\text { - Origen } \\
\text { urbanístico }\end{array}$ & $\begin{array}{l}\text { Información } \\
\text { geográfica } \\
\text { aplicada a } \\
\text { los datos } \\
\text { demográficos }\end{array}$ \\
\hline
\end{tabular}

Fuente: elaboración propia con datos del censo DANE 2005

En primer lugar, se consideran variables del nivel individual del jefe del hogar, el segundo es un nivel intermedio, circunscrito al ámbito del hogar y la vivienda. Para ambos se utilizaron los microdatos censales de 2005. En un tercer nivel de análisis, de ámbito barrial, se incluye, además del origen urbanístico presente desde el modelo anterior, la década en que se construyó el área urbana de cada polígono. Se busca incorporar la territarias 45 cohorte de construcción de los barrios, que sintetiza los efectos coyunturales del momento de la construcción y es una variable proxy de su edad, especialmente para los barrios informales en donde se viven pocos procesos de reemplazo del parque residencial por stock más nuevo (Jaramillo, 2012). Para su ello, la información geográfica tomada de Mayorga (2017) se aplicó a los datos demográficos de los hogares; la distribución de esta variable en la ciudad se presenta en la figura 2.

\section{¿Hay un efecto diferenciado de las variables que explican el allegamiento externo en cada zona de origen urbanistico?}

Para comparar el efecto de las variables que explican el allegamiento externo entre zonas de origen formal y aquellas de pasado informal que han sido legalizadas, se realiza un modelo por cada tipo de zona, incluyendo como variables independientes las que resultaron significativas en el modelo 1. Se eliminaron de este análisis los hogares que residen en zonas "aún informales" debido a que son escasas en la ciudad y a las dificultades en su análisis como colectivo, que no permite dar cuenta de un proceso de urbanización específico, en los términos que interesan a este trabajo.

Adicionalmente, para el modelo desarrollado con los asentamientos legalizados, se incluye el año de legalización, bajo la hipótesis de que en las prácticas de 
allegamiento no solo influye la cohorte de construcción del barrio y el origen de su urbanización, sino también el tiempo que ha transcurrido desde su incorporación al perímetro urbano, con sus respectivos efectos. La periodización del año de legalización se realizó siguiendo a Torres (2012), quien identifica tres momentos claves en la ciudad: a) La primera gran “amnistía urbana” en 1986, b) La amnistía de 1996 y c) La amnistía de 1998 y posteriores. Se produjo así, una variable que categoriza cinco tipos de sectores urbanos: los formales, los legalizados antes de 1986, los legalizados entre 1987 y 1996, los legalizados entre 1997 y 2006, y los que seguían siendo informales después de 2006, año que se toma como punto de referencia para lograr la correspondencia con los datos demográficos utilizados (2005).

\section{Resultados}

En Bogotá, según el Censo de 2005, el $22 \%$ de los hogares compartía su vivienda con otro. Cerca del $30 \%$ de los hogares que residían en los sectores de nivel socioeconómico (ICV) bajo presentaban esta condición, aunque no era exclusiva de estas zonas, ya que se presentaba en un $15 \%$ y $3 \%$ en las áreas de nivel socioeconómico medio y alto (figura 3 ).

Entre las zonas legalizadas de toda la ciudad (no solo las de ICV bajo), este porcentaje era de $30 \%$ y en las que se mantenían siendo informales era del $26 \%$
Figura 2. Sectores censales de Bogotá según década de construcción

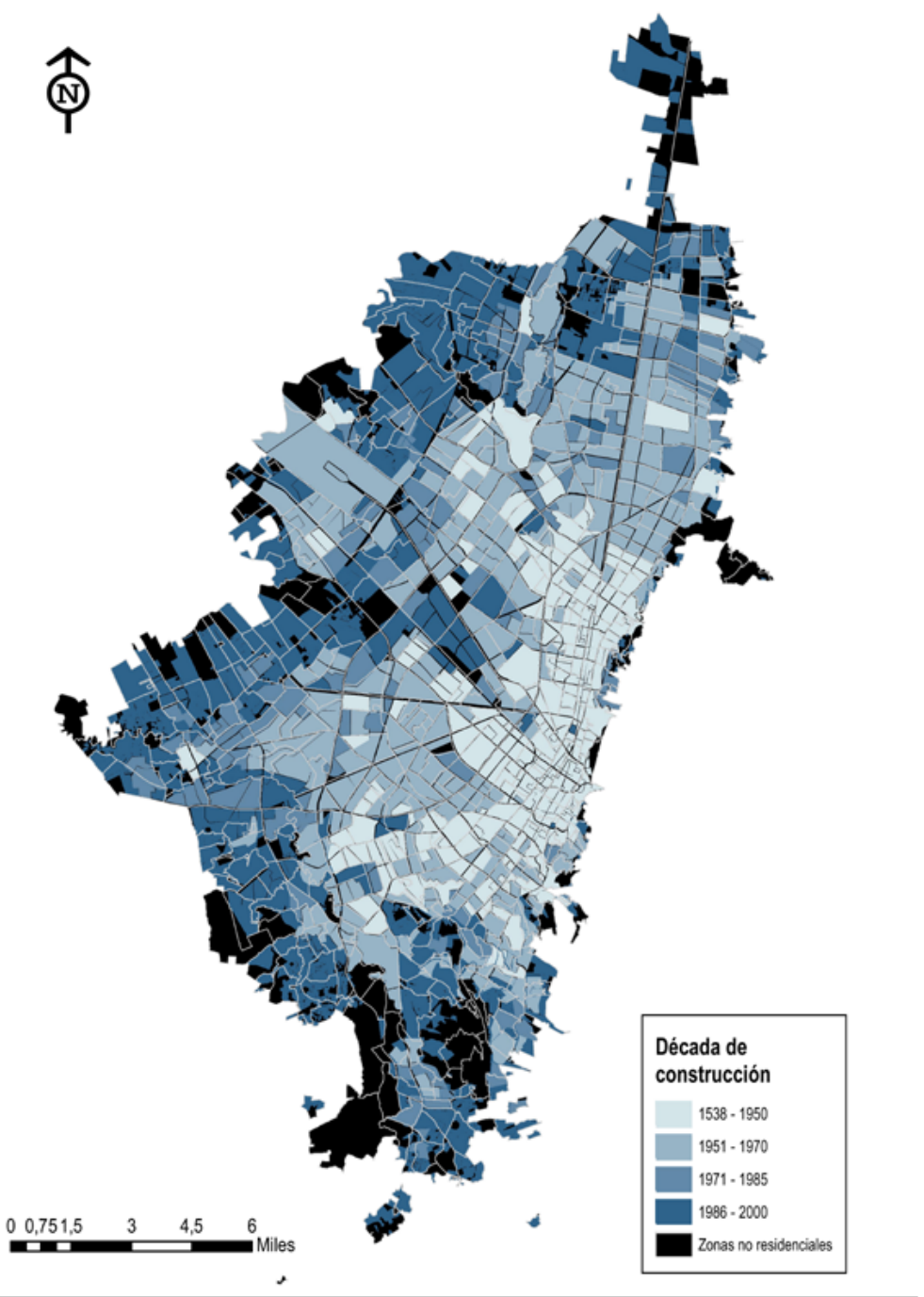

Fuente: elaboración propia con datos de Mayorga (2017).

(figura 4), presentándose un contraste con las zonas que siempre han sido formales, donde los hogares cohabitantes son el $17 \%$. territarias 45 
Figura 3. Distribución de hogares según cohabitación por nivel social del sector en que residen. Bogotá, 2005

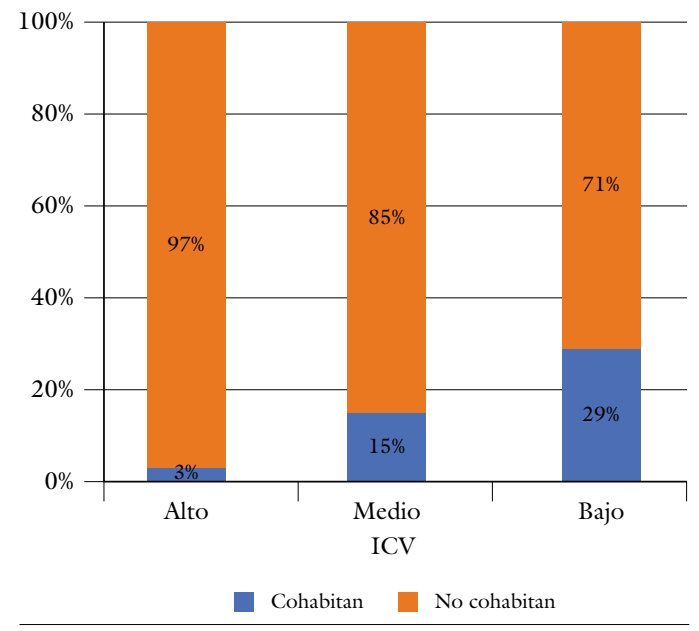

Fuente: elaboración propia con datos del censo DANE 2005.

Figura 4. Distribución de hogares según cohabitación por origen de urbanización de la zona de residencia. Bogotá, 2005

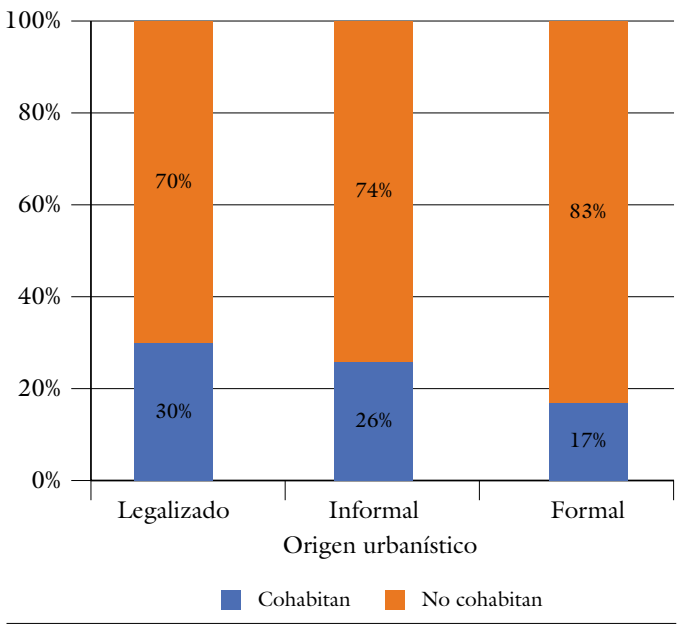

Fuente: elaboración propia con datos del censo DANE 2005.
Modelo 0: ¿Hay una relación estadística entre el origen urbanístico y la cohabitación?

Especificando en los sectores de nivel socioeconómico bajo, se observa que hay una mayor concentración de hogares que cohabitan en las zonas de pasado informal, ahora legalizadas, en donde alcanzan el $30 \%$. Este porcentaje es de $26 \%$ en las zonas aún informales y de $17 \%$ en las zonas siempre formales (figura 5).

Figura 5. Distribución de hogares según cohabitación por forma de urbanización de la zona en que residen. Sectores con ICV bajo Bogotá, 2005

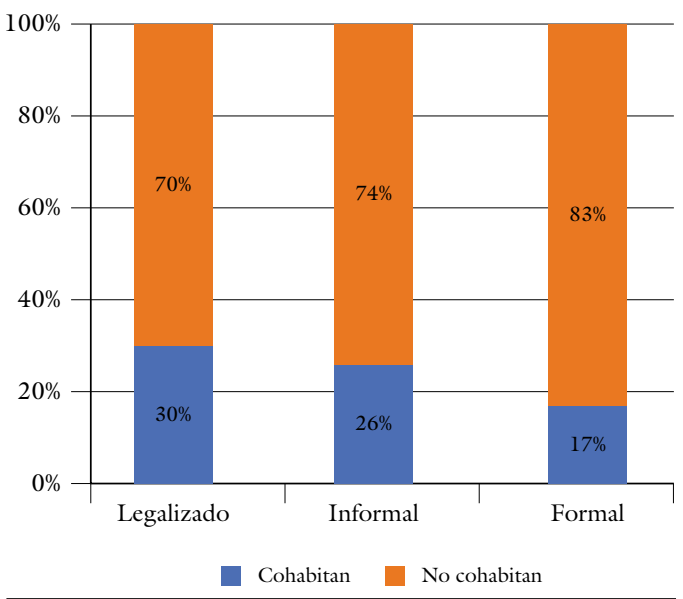

Fuente: elaboración propia con datos del censo DANE 2005.

Los resultados del modelo (tabla 2 ) confirman la relación estadística y la tendencia observada, ya que, al tomar como categoría de referencia a los hogares residentes en zonas siempre formales, se 
registra mayor propensión a la cohabitación de los hogares localizados en zonas legalizadas, seguidos por los que residen en zonas aún informales.

Tabla 2. Resultados Modelo 0. Cohabitación según origen urbanístico. Sectores con ICV bajo Bogotá, 2005

\begin{tabular}{|l|c|c|}
\hline \multicolumn{1}{|c|}{ Origen urbanístico } & Sig. & $\operatorname{Exp}(\mathbf{B})$ \\
\hline Legalizados & 0,000 & 1,382 \\
\hline Informales & 0,000 & 1,226 \\
\hline Formales (Ref) & 0,000 & 1 \\
\hline Constante & 0,000 & 0,341 \\
\hline
\end{tabular}

Nota: la bondad de ajuste del modelo proporciona un valor de Pseudo- $\mathrm{R}^{2}=0,39$, por lo que se considera aceptable. Fuente: elaboración propia con datos del censo DANE 2005.

\section{Modelo 1: Relación entre la} cohabitación y el origen urbanístico: ¿ efecto neto o de composición?

En lo que respecta a las variables explicativas del modelo, resumidas en la tabla 3 , la que no fue considerada como tal (pues no alcanzó el nivel de ganancia del $0,1 \%$ ), es el lugar de nacimiento del jefe del hogar. Es esperable que el efecto de la migración haya quedado subsumido en el del nivel educativo.

De las significativas, la primera es el tipo de vivienda, cuyo peso sobresale con respecto a las siguientes, que son la edad del jefe de hogar, el tipo de hogar, el nivel educativo del jefe, el sexo, y, por último, se ubican la década de construcción del barrio y el origen urbanístico. Así, subraya
Tabla 3. Resultados Modelo 1: Regresión por el método stepwise para la cohabitación según características individuales, del hogar, la vivienda y el origen urbanístico. Sectores con ICV bajo Bogotá, 2005

\begin{tabular}{|l|c|c|c|c|c|}
\hline & & $\begin{array}{c}-2 \text { Log } \\
\text { likelihood }\end{array}$ & Ganancia & $\begin{array}{c}\text { Porcentaje } \\
\text { de ganancia }\end{array}$ & $\begin{array}{c}\text { Chi } \\
\text { cuadrado }\end{array}$ \\
\hline Tipo de Vivienda & 1 & 1116689,49 & & 4,45 & 51986,276 \\
\hline Edad del jefe de hogar & 2 & 1104611,94 & 12077,550 & 1,09 & \\
\hline Tipo de hogar & 3 & 1099140,99 & 5470,950 & 0,50 & \\
\hline Nivel educativo jefe & 4 & 1094517,96 & 4623,023 & 0,42 & \\
\hline Sexo del jefe de hogar & 5 & 1092582,69 & 1935,279 & 0,18 & \\
\hline Década de construcción & 6 & 1087998,82 & 1908,354 & 0,18 & \\
\hline Origen urbanístico & 7 & 1090849,33 & 1733,357 & 0,16 & \\
\hline $\begin{array}{l}\text { Jefe no nacido } \\
\text { en Bogotá }\end{array}$ & 8 & 1090391,44 & 457,889 & 0,04 & \\
\hline
\end{tabular}

Fuente: elaboración propia con datos del censo DANE 2005

que a pesar de la preponderancia de las variables de vivienda y sociodemográficas, al controlarlas, el modelo revela que existe un efecto neto del origen urbanístico en la propensión a la cohabitación.

$\mathrm{Al}$ incluir estas variables en una regresión logística se obtiene el modelo presentado en la tabla 4 . Los resultados para cada variable se describen a continuación.

La importancia jerárquica del tipo de vivienda, así como la diferencia entre las odds ratio de sus categorías, revelan la fuerte asociación de la cohabitación con las condiciones de infraestructura necesarias para que suceda. En primer lugar, es evidente que reúne características como una mayor posibilidad de modificación y ampliación, y más disponibilidad de 
Tabla 4. Resultados Modelo 1: Regresión logística para la cohabitación según características individuales, del hogar, la vivienda y la biografía del barrio. Sectores con ICV bajo Bogotá, 2005

\begin{tabular}{|c|c|c|c|c|}
\hline & & B & Sig. & $\operatorname{Exp}(b)$ \\
\hline \multirow{3}{*}{ Tipo de vivienda } & Casa & ,986 & 0,000 & 2,681 \\
\hline & Otro &,- 704 & 0,000 & , 495 \\
\hline & Apartamento & & & 1 \\
\hline \multirow{4}{*}{ Edad } & Menor de 25 & ,872 & 0,000 & 2,393 \\
\hline & Entre 26 y 40 & ,421 & 0,000 & 1,524 \\
\hline & Entre 41 y 60 &, 041 & 0,294 & 1,042 \\
\hline & Más de 60 (Ref) & & & 1,000 \\
\hline \multirow{5}{*}{ Tipo de hogar } & Unipersonal & ,114 & 0,000 & 1,121 \\
\hline & Nuclear (Ref) & & & 1,000 \\
\hline & Extenso &,- 443 & 0,000 &, 642 \\
\hline & Compuesto & ,083 & 0,000 & 1,087 \\
\hline & Sin núcleo &,- 361 & 0,000 & ,697 \\
\hline \multirow{3}{*}{ Educación } & Primaria & ,491 & 0,000 & 1,634 \\
\hline & Secundaria & ,368 & 0,000 & 1,444 \\
\hline & Superior (Ref) & & 0,000 & 1 \\
\hline \multirow{2}{*}{ Sexo } & Hombre &,- 229 & 0,000 & ,795 \\
\hline & Mujer & & 0,000 & 1 \\
\hline \multirow{4}{*}{ Década desarrollo } & Hasta 1960 & ,276 & 0,000 & 1,317 \\
\hline & Década 60 y 70 & , 137 & ,000 & 1,147 \\
\hline & Década 80 & ,031 &, 000 & 1,032 \\
\hline & Década 90 y 2000 (Ref) & & & 1,000 \\
\hline \multirow{3}{*}{ Origen Urbanístico } & Legalizados hasta 1986 & ,266 & 0,000 & 1,304 \\
\hline & Informales &,- 143 &, 000 & ,867 \\
\hline & Siempre formales (Ref) & & 0,000 & 1 \\
\hline
\end{tabular}

Nota: la bondad de ajuste del modelo proporciona un valor de Pseudo- $\mathrm{R}^{2}=0,42$ por lo que se considera aceptable. Fuente: elaboración propia con datos del censo DANE 2005. 
espacio, que, sin embargo, ha ido disminuyendo con el tiempo y tiene contrastes entre los niveles sociales (Secretaría Distrital de Planeación, 2013). Este hallazgo se inserta en el proceso de convergencia hacia el apartamento que vive la ciudad, (García-García \& Módenes, 2018) concentrado principalmente en el centro expandido, en donde muchas de las casas que persisten están mutando a otros usos (oficinas, hoteles y otros servicios) (UAECD, 2012; De Urbina, 2013; Salazar et al., 2015). En cambio, en los sectores socioeconómicos bajos, para 2005 la casa seguía aportando cerca del $50 \%$ del stock residencial, concentrando el $62 \%$ de las casas de la ciudad, lo que facilita la comprensión del fenómeno del allegamiento externo.

La segunda variable en importancia fue la edad del jefe del hogar, que confirma que la cohabitación es un fenómeno altamente demográfico, que aglutina las altas propensiones antes de los 40 años, con su auge en hogares con jefes menores de 25 años, $40 \%$ de los cuales cohabita con otro hogar. La sobrerrepresentación en etapas iniciales de la vida adulta o familiar, muestra que la cohabitación facilita el proceso de emancipación y que va disminuyendo con el paso de la vida, cuando se espera un ascenso gradual en la carrera laboral y, con ello, mejorar la capacidad de pago de un espacio independiente.

Sin embargo, el diferencial en la propensión al fenómeno por rangos de edad, muestra que no hay una correspondencia bilateral entre hogares mayores que reciben allegados y hogares jóvenes que son acogidos, siendo ambos catalogados como cohabitantes. La sobrerrepresentación de estos últimos revela que, por un hogar receptor adulto hay varios hogares allegados jóvenes. Esta situación resulta de difícil exploración por la forma de recolección censal de datos, que no indaga por el hogar principal y el (los) secundario(s) al interior de la vivienda. Tampoco se indaga por la relación o parentesco entre los miembros de los hogares y el estatus de tenencia de la vivienda de cada uno, lo que permitiría comprender mejor el fenómeno.

Según la variable de tipo de hogar, aunque las diferencias son mínimas, la propensión al allegamiento externo aumenta ligeramente para los hogares unipersonales y compuestos (con no familiares que cohabitan); en estos casos, la frontera con el allegamiento externo es muy fina y depende de las formas de clasificación de los hogares. A partir de los resultados de la variable educativa, se confirma que son los menos educados los que más propensión tienen a la cohabitación, lo cual es esperable debido a que se trata de una variable de diferenciación socioeconómica. Por último, la variable sexo muestra mayor propensión de los hogares de jefatura femenina, los cuales son reconocidos por tener estructuras familiares más precarias y propensas a hacer uso de las redes de apoyo que provee la cohabitación. territarias 45 179 
Las variables que exploran la biografía del barrio revelan varios elementos. De un lado, al analizar el efecto del año de desarrollo urbano sobre la cohabitación de los hogares, se observa que las odds ratio aumentan levemente en función de la antigüedad del proceso de urbanización, lo que revela que, aun controlando por el tipo de urbanización, existe un efecto de generación de los barrios. Lo anterior puede asociarse con prácticas antiguas de cohabitación que se mantienen y reproducen en infraestructuras que así lo permiten.

De otra parte, llegando a nuestro principal foco de interés que es el origen urbanístico (tabla 5), en este modelo se confirma la mayor propensión a la cohabitación en las zonas que fueron producidas informalmente y luego legalizadas, frente a aquellas siempre formales, relación que ya había sido revelada en el modelo 0 . Es posible establecer entonces, que la mayor cohabitación en las antiguas áreas informales no solo se debe a un efecto de composición socioeconómica y residencial de las mismas, sino que tiene que ver con los elementos propios de este tipo urbanístico referidos, por ejemplo, a la forma de producción de la vivienda de la informalidad.

En cambio, los resultados para los hogares residentes en zonas aún informales, sí cambiaron entre el modelo $0 \mathrm{y}$ el 1 ; reduciéndose en este caso las odds ratio con respecto a la categoría de referencia. Lo anterior puede asociarse con un fuerte efecto de composición de estas zonas, que, como se dijo anteriormente, son escasas y altamente diversas.

Tabla 5. Resultados Modelo 0 y 1 de cohabitación para la variable independiente origen urbanístico. Sectores con ICV bajo Bogotá, 2005

\begin{tabular}{|l|c|c|c|c|}
\hline \multirow{2}{*}{} & \multicolumn{2}{|c|}{ Modelo 0 } & \multicolumn{2}{c|}{ Modelo 1 } \\
\cline { 2 - 5 } & Sig. & $\operatorname{Exp}(\mathbf{B})$ & Sig. & $\operatorname{Exp}(\mathbf{B})$ \\
\hline Legalizados & 0,000 & 1,382 & 0,000 & 1,304 \\
\hline Informales & 0,000 & 1,226 & 0,000 & 0,866 \\
\hline Formales (Ref) & 0,000 & 1 & 0,000 & 1 \\
\hline Constante & 0,000 & 0,341 & 0,000 & 0,120 \\
\hline
\end{tabular}

Fuente: elaboración propia con datos del censo DANE 2005.

\section{Modelo 3 ¿Varía el efecto de las variables que inciden en la cohabitación en función del origen urbanístico de las zonas en que residen los hogares?}

Al realizar dos modelos que comparan sus efectos en las zonas legalizadas y en las formales, tomando estos últimos como grupo de control, es posible dilucidar cómo opera el efecto del origen urbanístico. La principal diferencia entre los dos modelos se reveló con los resultados del método stepwise, que entrega la jerarquía en la que las variables aportan a la explicación de la cohabitación en cada caso, descartando a las que no superan el 0,1 de ganancia en la explicación del fenómeno. 
Como se observa en la tabla 6 , el orden y el porcentaje de aporte de las variables en los tres modelos revelan similitudes y diferencias, que muestran la diversidad al interior de los sectores populares (ICV bajo) según el origen urbanístico. Dentro de las similitudes, sobresale que las dos variables que siempre mantienen los primeros lugares jerárquicos son el tipo de vivienda y la edad del jefe, confirmándose que la cohabitación es una práctica con alto contenido demográfico, posibilitada por una infraestructura flexible. Asimismo, en todos los modelos fue eliminado el origen migratorio del jefe del hogar por no tener un aporte significativo a la explicación de la cohabitación.
Pasando a las diferencias, se observa que, en el modelo que describe los contextos de urbanización formal, se mantuvieron todas las variables incluidas en el modelo general, mostrando que hay una alta heterogeneidad en la tendencia a la cohabitación en estas zonas. En este caso, la década de desarrollo urbano escaló al tercer lugar, cuando en el modelo general fue ubicada como la última en importancia. El poder explicativo del tiempo supera al de las variables sociales y se expresa a través de un efecto de cohorte de desarrollo de los barrios, que implica la exposición a patrones urbanísticos y de planeación propios de una época.

En cambio, en las zonas legalizadas, el modelo mostró que solo cuatro variables

Tabla 6. Resultados regresión por el método stepwise para la cohabitación según características individuales, del hogar, la vivienda y la biografía del barrio. ICV bajo Bogotá, 2005

\begin{tabular}{|l|c|c|c|c|c|c|}
\hline \multirow{2}{*}{\multicolumn{1}{|c|}{ Variables }} & \multicolumn{2}{|c|}{ Modelo I ICV bajo total } & \multicolumn{2}{c|}{ Formal ICV bajo } & \multicolumn{2}{c|}{ Legalizado ICV bajo } \\
\cline { 2 - 7 } & $\begin{array}{c}\text { Lugar de } \\
\text { aporte al } \\
\text { modelo }\end{array}$ & $\begin{array}{c}\text { Porcentaje } \\
\text { de ganancia }\end{array}$ & $\begin{array}{c}\text { Lugar de } \\
\text { aporte al } \\
\text { modelo }\end{array}$ & $\begin{array}{c}\text { Porcentaje } \\
\text { de ganancia }\end{array}$ & $\begin{array}{c}\text { Lugar de } \\
\text { aporte al } \\
\text { modelo }\end{array}$ & $\begin{array}{c}\text { Porcentaje ganancia } \\
\text { de }\end{array}$ \\
\hline Tipo de Vivienda & 1 & 4,45 & 1 & 3,43 & 1 & 4,87 \\
\hline Edad del jefe de hogar & 2 & 1,09 & 2 & 0,88 & 2 & 1,18 \\
\hline Tipo de hogar & 3 & 0,5 & 5 & 0,57 & 3 & 0,49 \\
\hline Nivel educativo jefe & 4 & 0,42 & 4 & 0,74 & - & - \\
\hline Sexo del jefe de hogar & 5 & 0,18 & 6 & 0,13 & 4 & 0,21 \\
\hline Década de desarrollo & 6 & 0,18 & 3 & 0,82 & - & - \\
\hline Origen urbanístico & 7 & 0,16 & - & - & - & - \\
\hline
\end{tabular}

Fuente: elaboración propia con datos del censo DANE 2005. 
aportaron más del 0,1 de ganancia, por lo que se considera que son las que tienen un poder explicativo sobre la cohabitación. Se trata del tipo de vivienda, la edad del jefe del hogar, el tipo de hogar y el sexo. Se revela así, que esta es una estrategia que cobija a las estructuras familiares frágiles e iniciales en el ciclo vital.

Con todo, las variables excluidas del modelo también aportan información valiosa. De un lado, que el nivel educativo no resultara significativo estadísticamente, evidencia que contar con mayor educación en las áreas legalizadas no protege de la tendencia a la cohabitación. La no operación de un filtro socioeconómico en el fenómeno plantea preguntas sobre los procesos de movilidad social que produce la educación y sus implicaciones en las condiciones de vida en las áreas legalizadas. También genera interrogantes sobre la aceptación cultural que existe sobre la cohabitación, que hace que, en las zonas de origen informal, no sea exclusiva de los menos educados (o, por extensión, de los más pobres).

Por otro lado, sobresale el poco poder explicativo de las variables biográficas del barrio, referidas al año de legalización y de desarrollo urbano. En cuanto al efecto de la legalización, es imposible determinar si fue común a todos los barrios sin importar su temporalidad, o si la intervención estatal no impacta las prácticas de corresidencia, ya que las transformaciones que acarrea la legalización se asocian con la llegada de Programas de Mejoramiento
Barrial, que se concentran en el nivel meso del hábitat, es decir, sobre los espacios públicos y vías, generando dotación de infraestructura y equipamientos. Esto, sumado al poco efecto del tiempo de desarrollo del barrio sobre la cohabitación, muestra que esta es inherente a la forma en que se produce y habita la vivienda desde la lógica informal, la cual se mantiene estática ante las transformaciones estatales o los efectos de cohorte urbanistica. Puede entenderse como una marca de la informalidad que permanece a pesar de la transición a la formalidad en otras dimensiones del hábitat.

Los resultados de las odds ratio para cada variable en los dos modelos se presentan en la tabla 7.

Aunque en todas las categorías de las variables se mantienen las odds ratio observadas en el modelo 0 , es posible enfatizar en el poder explicativo de la casa sobre el allegamiento. Cabe recordar que la ciudad vivió un proceso acelerado de cambio tipológico de la casa al apartamento en el período 1993-2005, lo cual conduce a indagar por las formas de uso de las casas subsistentes. Este interrogante puede responderse de manera distinta para las zonas formales y las legalizadas.

En las zonas formales, esta información debe ser leída conjuntamente con los resultados de la variable del año de desarrollo urbano, que mostró mayor propensión a la cohabitación en las zonas más antiguas y una tendencia decreciente. Lo anterior puede revelar la persistencia 
Tabla 7. Resultados Regresión logística para el allegamiento externo según características individuales, del hogar, la vivienda y la biografía del barrio. Sectores formales con ICV bajo Bogotá, 2005

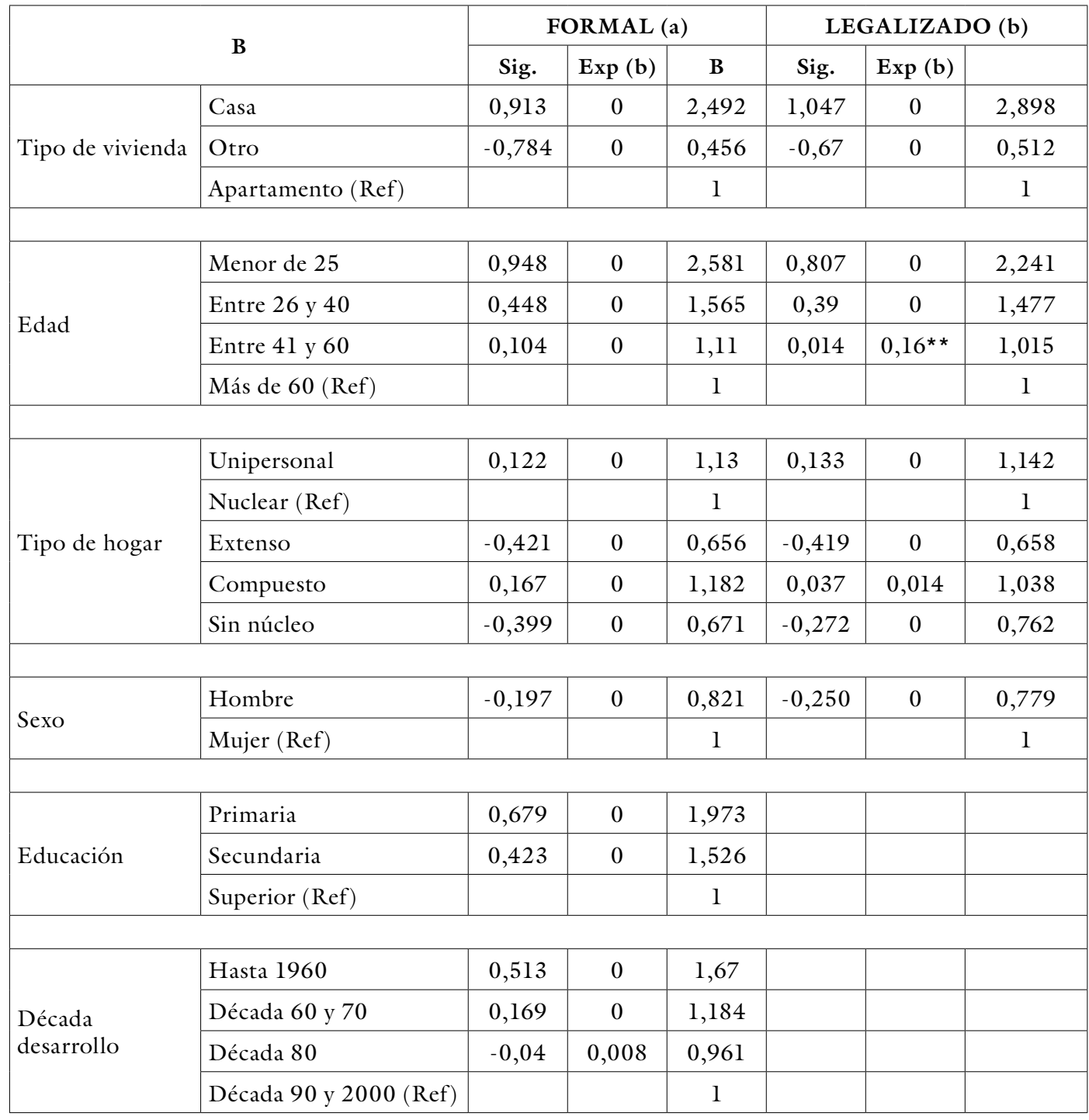

Nota: a) La bondad de ajuste del modelo proporciona un valor de Pseudo- $\mathrm{R}^{2}=0,43$, por lo que se considera aceptable. b) La bondad de ajuste del modelo proporciona un valor de Pseudo-R2 = 0,4l, por lo que se considera aceptable.

Fuente: elaboración propia con datos del censo DANE 2005. 
de formas de cohabitación también antiguas, permitidas por la transformación de espacios interiores de las casas para usos residenciales. Esta práctica tiene como antecedente el fenómeno de los inquilinatos, que fueron una forma de hábitat popular y una estrategia de supervivencia familiar, usada en el auge del éxodo rural a la ciudad. Se localizaban principalmente en el centro de la ciudad (Bonvalet \& Dureau, 2002) y constituían un mercado de subarriendo informal de habitaciones para el uso de hogares, que compartían espacios de baño y cocina en condiciones de precariedad y hacinamiento. Estas prácticas de formalidad antigua parecieran similares a las alojadas en la informalidad actual.

En las zonas informales, la preponderancia de la casa se explica porque la vivienda es autoconstruida por sus posteriores usuarios (Bazant, 1985). La expansión de la vivienda incrementa los espacios disponibles para ser ocupados por otros hogares, familiares o no, que pueden participar del mercado de arrendamiento (Jaramillo, 2008). Dicha "libertad urbanística" se constituye en una ventaja comparativa frente al mercado formal de vivienda social, que provee infraestructuras estáticas e inmodificables, que soslayan las prácticas de cohabitación de los sectores populares.

En suma, es posible decir que en las zonas legalizadas se registra una mayor homogeneidad en la presencia de la cohabitación, que sucede independientemente de las variables biográficas del barrio, o del nivel social, presentándose como un recurso para la emancipación de hogares vulnerables. Por el contrario, en los barrios formales, hay más heterogeneidad - aunque menor presencia- del fenómeno, pues este depende tanto de condiciones de diferenciación social, de composición y momento de la vida de los hogares, como de la historia del barrio.

\section{Conclusiones}

Se comprobó que la informalidad tiene un poder explicativo en las diferencias que hay entre las prácticas residenciales de los hogares pobres. La forma en que opera el mercado informal, deja una marca difícil de transformar en el tiempo, generando un contexto urbano diferenciado. La principal causa por la que esto sucede es el uso de la autoconstrucción y el desarrollo progresivo de la vivienda, que asume su provisión como un proceso, tan flexible y dinámico como lo es la composición de los hogares. A su vez, genera unas condiciones de posibilidad física para la cohabitación, en un contexto donde las redes de solidaridad tienen un peso muy alto dentro de la provisión del bienestar.

Sin embargo, es posible pensar este problema a partir del continuo formalinformal que pretende matizar la dicotomía entre estos dos conceptos. Este desafío fue abordado al incorporar las variables biográficas de los barrios de origen informal, explorando cómo han incidido los procesos históricos previos en 
la disposición actual de la cohabitación. El resultado mostró que las diferencias en los momentos de las intervenciones estatales y en el tiempo transcurrido desde el inicio del barrio, no inciden en los niveles de cohabitación observados. Esto confirma que a pesar de la formalización de elementos físicos que podrían conducir a la formalización de los barrios, el diferencial en las prácticas residenciales permanece. Una parte de este fenómeno se explica por el arraigo cultural de la cohabitación y por la persistencia de las dificultades en el acceso a la vivienda, otra parte puede explicarse porque la cohabitación sigue constituyendo una ventaja que ofrecen las infraestructuras residenciales existentes.

La persistencia de la cohabitación obliga a repensar la relación entre hogares y viviendas, así como su uso en las políticas públicas en el contexto latinoamericano, demostrando, por ejemplo, que la formación de hogares entre quienes viven en zonas de origen informal, no está determinada por la disponibilidad de viviendas. Estudios más profundos al respecto son necesarios para desentrañar la compleja relación entre intimidad y apoyo en la convivencia entre hogares, así como sus implicaciones en las definiciones del déficit cuantitativo de viviendas.

Una segunda forma de abordar el continuo, fue analizar el fenómeno también en la formalidad. Los resultados mostraron la importancia de las variables temporales en la presencia de la cohabitación, lo que conduce a pensar que la permanencia o envejecimiento de las casas producen prácticas cercanas a las existentes en los contextos de informalidad.

Por último, es preciso señalar que los usos de estos hallazgos son aplicables a la política pública en dos sentidos. En primer lugar, resaltan el componente demográfico como un argumento de conveniencia para la "escogencia" de la informalidad, dado que las complejas prácticas familiares que esta permite no son tenidas en cuenta en el diseño de viviendas sociales destinadas a los grupos sociales más pobres. En segundo lugar, el reconocimiento de dicha composición residencial resulta de vital importancia en la interacción con la ciudadanía, en el marco de la intervención estatal, para el mejoramiento las condiciones de vida de la informalidad.

\section{Referencias}

Abramo, P. (2012). La ciudad com-fusa: mercado y producción de la estructura urbana en las grandes metrópolis latinoamericanas. Eure 38(114), 3569. https://doi.org/10.4067/S025071612012000200002

Bazant, J. (1985). Autoconstrucción de vivienda popular. Trillas.

Blanco-Pepi, M. M., Cacopardo, G., \& Freire, P. G. (2017). Aportes a la gestión del hábitat popular. Investigación+Acción, (20), 11-38.

Bonvalet, C., \& Dureau, F. (2002). Los modos de habitar: unas decisiones 
condicionadas. En F. Dureau, V. Dupont, E. Lelievre, J. Lévy, \& T. Lulle (Eds.), Metrópolisen movimiento: una comparación internacional (pp. 6987). Alfaomega.

Camargo, A., \& Hurtado, A. (2012). Informalidad del siglo XXI. Características de la oferta informal de suelo y vivienda en Bogotá durante la primera década del siglo XXI. Territorios, (27), 71-104.

Camargo, A., \& Hurtado, A. (2013). Urbanización informal en Bogotá: agentes y lógicas de producción del espacio urbano. Revista INVI, 28(78), 77-108.

De Urbina, A. (2013). El Centro Histórico de Bogotá "de puertas para adentro": ¿ el deterioro del patrimonio al servicio de la gentrificación? Cuadernos De Vivienda y Urbanismo, 5(9), 46-69.

Di Virgilio, M. M., \& Gil, M. L. (2012). Estrategias habitacionales de familias de sectores populares y medios residentes en el área metropolitana de Buenos Aires (Argentina). Revista de Estudios Sociales, (44), 158-170.

García García, D. M., \& Módenes, J. A. (2018). Transiciones demográficas, nuevas formas residenciales y segregación social: transformaciones recientes del espacio urbano de Bogotá. Notas de población, 45(106), 217-250

Gelabert, D., \& González, D. (2013, eneroabril). Progresividad y flexibilidad en la vivienda. Enfoques teóricos. Arquitectura y Urbanismo, 34(1), 17-31.
González, M. (2001). From the resources of poverty to the poverty of resources? The erosion of a survival model. Latin American Perspectives, 28(4), 72-100. Jaramillo, S. (2008). Reflexiones sobre la "informalidad" fundiaria como peculiaridad de los mercados del suelo en las ciudades de América Latina. Territorios (18-19), 11-53.

Jaramillo, S. (2012). Urbanización Informal: Diagnósticos y políticas. Una revisión al debate latinoamericano para pensar líneas de acción actuales. Documentos CEDE. http://hdl.handle. net $/ 1992 / 8320$

Jelin, E. (2005). Las familias latinoamericanas en el marco de las transformaciones globales: hacia una nueva agenda de políticas públicas. En I. Arriagada. (Ed.), Politicas hacia las familias, protección e inclusión sociales (pp. 69-88) [Serie Seminarios y Conferencias No. 46]. CEPAL.

Jovell, A. J. (1995). Análisis de regresión logistica. Centro de Investigaciones Sociológicas.

López, W. (2017). La recomposición social $y$ urbana del territorio como alternativa a los programas de mejoramiento barrial. Caso de estudio: Bogotá (Tesis doctoral). Universidad Tecnológica de la Habana "José Antonio Echeverría", La Habana, Cuba.

Mayorga, M. (2017). El proceso de urbanización de los humedales de Kennedy en Bogotá-Colombia: Una perspectiva 
normativa. Papeles de Coyuntura, (42), 14-53.

Mayorga, M., García-García D. M., \& Barrera R. (2019). Cálculo de un indicador de calidad de vida básico para Bogotá por secciones censales mediante análisis factorial. Perspectiva Geográfica, 24(1). https://doi. org/10.19053/01233769.7861

Salazar, C., Contreras, Y., Dureau, F., \& Le Roux, G. (2015). Patrones de poblamiento de Bogotá y Santiago de Chile en los albores del siglo XXI. En F. Dureau, T. Lulle, S. Souchaud, \& Y. Contreras (Eds), Movilidades y cambio urbano. Bogotá, Santiago y Sao Paulo (pp. 99-126). Universidad Externado de Colombia.

Secretaría Distrital de Planeación. (2013). Índice de calidad de la vivienda en Bogotá. Ciudad de Estadisticas [Boletín No. 60]. http://www.planeacionbogota.gov.co/sites/default/ files/dicel44-boletinindcondvivienda-04122013_0.pdf

TECHO. (2015). Derecho a Bogotá. Informe de asentamientos informales. https:// issuu.com/techocolombia/docs/ derecho_a_bogot_-_ 40
Torres, C. (2009). Ciudad informal colombiana: barrios construidos por la gente. Universidad Nacional de Colombia

Torres, C. (2012). Legalización de barrios: acción de mejora o mecanismo de viabilización fiscal de la ciudad dual. Bulletin de l'Institut français d'études andines, 41(3), 441-471.

Ullmann, H., \& Valera, C. (2014). La evolución de las estructuras familiares en América Latina, 1990-2010: Los retos de la pobreza, la vulnerabilidad y el cuidado [Serie Políticas Sociales]. CEPAL.

Unidad Administrativa Especial de Catastro Distrital (UAECD). (2016) Estadisticas catastrales. https://www. catastrobogota.gov.co/recurso/resultados-censo-inmobiliario-2012

UN-Hábitat. (2004). Hábitat y desarrollo urbano. CENAC

Urrutia, J., Jirón, P., \& Lagos, Á. (2016). ¿Allegamiento o co-residencia? de un fenómeno problemático a una solución innovadora para atender el déficit habitacional crónico. Revista $C / A$, 152, http://repositorio.uchile.cl/handle/2250/139799 